\title{
Application of Probabilistic Sensitivities to Probabilistic Fatigue Analysis of Gas Turbine Engine Disks
}

\author{
R. Wesley Osborn ${ }^{*}$, Harry R. Millwater ${ }^{\dagger}$ \\ University of Texas at San Antonio, San Antonio, Texas 78249
}

\begin{abstract}
A methodology is presented and applied that determines the sensitivities of the probability-offracture of a gas turbine disk fatigue analysis with respect to the parameters of the probability distribution of the random variables. This information is useful in determining the importance of the random variable parameters and the relative ranking of the variables. The methodology is demonstrated using a probabilistic fatigue analysis of a gas turbine compressor disk using the computer program DARWIN ${ }^{\circledR}$. Although originally intended for use with titanium alloys, DARWIN has been extended to allow analyses to be conducted on other common materials used in the production of gas turbine disks that contain high-frequency anomalies. This paper takes into account this new capability and provides a systematic technique for computing sensitivities in a zone-based probabilistic framework.
\end{abstract}

\section{Nomenclature}

$a \quad=$ defect size

$a_{\text {min }}=$ minimum defect size in exceedance curve

$a_{\max }=$ maximum defect size in exceedance curve

$\mathrm{cov}=$ coefficient of variation

$E[$ ] = expected value operator

$f_{\widetilde{x}}(\widetilde{x})=$ joint density function of vector of random variables $\widetilde{x}$

$f_{A}(a)=$ probability density function of the exceedance curve

$g=$ limit state function

$I \quad=$ indicator function

$\lambda=$ mean anomaly occurrence rate

$N(a)=$ exceedance value associated with initial crack size

$P \quad=$ general probability-of-fracture

$P_{M C} \quad=$ probability-of-fracture computed from Monte Carlo sampling

$P_{F, \text { zone } k}=$ probability-of-fracture for a defect originating within zone $k$

$P_{F} \quad=$ probability-of-fracture of disk

$\Psi=$ volume of component or surface area

$\theta_{i} \quad=$ statistical parameter of probability density function associated with variable $x_{i}$

$\widetilde{x} \quad=$ vector of random variables, $\tilde{x}=\left(x_{1}, x_{2}, \ldots\right)$

$\tilde{X} \quad=$ median value of random variable $X$

\footnotetext{
* Graduate Student, Department of Mechanical Engineering and Biomechanics, 6900 N. Loop 1604 W. San Antonio, Texas, AIAA Student Member

${ }^{\dagger}$ Assistant Professor, Department of Mechanical Engineering and Biomechanics, 6900 N. Loop 1604 W. San Antonio, Texas, AIAA Member
} 


\section{Introduction}

In 1990, the FAA released a post-crash report after a plane crash in Sioux City Iowa in 1989 recommending an initiative to develop a probabilistic damage tolerance approach to reduce the risk of reoccurrence ${ }^{1}$. In 1995 , the FAA established a grant to Southwest Research Institute, General Electric, Honeywell, Pratt \& Whitney, and RollsRoyce to develop a probabilistically-based damage tolerance design code to augment the current safe-life approach for life management of commercial aircraft gas turbine rotors and disks ${ }^{2}$. This grant led to the development of the computer program DARWIN (Design Assessment of Reliability With INspection). The program is capable of computing the probability-of-fracture of a component as a function of flight cycles, considering random defect occurrences and location, and random inspection schedules as shown in Table $1^{3,4}$.

Table 1. Random Variables Considered in DARWIN

\begin{tabular}{|l|}
\hline Defect Distribution \\
\hline Probability of Detection \\
\hline Time of Inspection \\
\hline Finite Element Stress (multiplier) \\
\hline Life Scatter (fracture mechanics) \\
\hline
\end{tabular}

In DARWIN, the random variables are characterized, statistically, by a variety of distributions and these distributions by various parameters. The probability-of-fracture is computed by Monte Carlo or Importance Sampling probabilistic methods. The distribution parameters for these random variables can have a significant effect on the probability-of-fracture. These distribution parameters are obtained though various testing, evaluation, and statistical methods, which can be costly to perform, hence the need to develop a method for evaluating how sensitive the probability-of-fracture is to a small change in the value of these parameters and to determine the importance of the parameters to the probability-of-fracture estimate.

This paper is focused on demonstrating the methodology and application of probabilistic sensitivities in the probabilistic fatigue computer program DARWIN. The methodology used to obtain the probabilistic sensitivities for the defect distribution, Life Scatter, and Stress Multiplier random variables using Monte Carlo sampling. The variables Life Scatter and Stress Multiplier are defined by the lognormal distribution. However, this methodology is capable of determining the sensitivity of the probability-of-fracture to parameters of many different distribution types (e.g. Normal, Truncated Normal, Weibull, Extreme Value, Uniform, Triangular, Two-Parameter Beta, Gamma, Lognormal and Exponential). In addition, the capability to compute sensitivities with respect to the bounds of the defect distribution, defined by an exceedance curve is also presented.

\section{Probabilistic Methodology in DARWIN}

The computer program DARWIN employs a "zone-based" system reliability methodology to compute the probability-of-fracture of a disk as a function of flight cycles ${ }^{5}$. The disk is discretized into a number of zones in which each sub-region of a zone generally has a uniform stress state, the same properties, and anomaly distribution ${ }^{2}$. In other words, the risk computed for each sub-region of material in the zone will be the same; thus, the material is grouped into a zone ${ }^{2}$.

Originally, DARWIN was developed for titanium materials, where inherent anomalies may be present in the form of brittle alpha phase particles that are assumed to form growing cracks during to first cycle of applied $\operatorname{load}^{6}$. However, the occurrence rate of these particles is very small, therefore the failure probability of the component can be approximated by taking the sum of the failure probabilities of zones ${ }^{6}$. In contrast, other materials may exhibit a larger number of anomalies that are either a result of manufacturing or inherently associated with the material processing ${ }^{6}$. When considering these materials, a single zone could contain a number of anomalies with unequal crack formation periods requiring an extension to the probabilistic method ${ }^{6}$. To address this issue, a new probabilistic method has been developed based on the original zone-based framework developed for titanium alloys $^{6}$. The sensitivities demonstrated here are capable of dealing with both anomalies associated with the volume and surface of the volume. The equations are developed such that the sensitivity of the probability-of-fracture to the parameters of the random variable distributions can be determined as a by-product of the analysis, that is, no additional fracture mechanics calculations are required. 


\section{A. Probabilistic Treatment of Multiple Anomalies ${ }^{6}$}

The probability-of-fracture within an individual zone, $P_{F, z o n e}$, can be computed as

$$
P_{F, \text { zone }}=\sum_{i=1}^{\infty} P(i \text { anomalies }) \cdot P(\text { fracture } \mid i \text { anomalies })
$$

where

$$
P(i \text { anomalies })=\frac{\exp (-\lambda) \cdot \lambda^{i}}{i !}
$$

is the probability of occurrence of $i$ anomalies, $\lambda$ is the anomaly occurrence rate, and

$$
\begin{gathered}
P(\text { fracture } \mid i \text { anomalies })=1-P(\text { no fracture } \mid i \text { anomalies }) \\
=1-\prod_{j=1}^{i} P(\text { no fracture } \mid \text { anomaly } j)
\end{gathered}
$$

is the probability-of-fracture given that $i$ anomalies are present.

The probability-of-fracture for a given number of cycles, $N_{o}$, is defined as

$$
P(g \leq 0)=P\left(N_{f} \leq N_{o}\right)
$$

where $g$ denotes the limit state, $N_{f}$ the computed cycles-to-failure, and $N_{o}$ the user-specified number of cycles.

Therefore,

$$
P(\text { no fracture } \mid \text { anomaly } j)=P(g>0)
$$

Eq. ( 3 )can be expressed as:

$$
P(\text { fracture } \mid i \text { anomalies })=1-[P(g>0)]^{i}
$$

Inserting Eqs. ( 2 )and ( 7 ) into Eq.( 1 ):

$$
P_{F, \text { zone }}=\sum_{i=1}^{\infty} \frac{\exp (-\lambda) \cdot \lambda^{i}}{i !} \cdot\left[1-(P(g>0))^{j}{ }_{-}^{-}\right.
$$

or

$$
P_{F, \text { zone }}=1-\exp [-\lambda(1-P(g>0))]
$$


or

$$
P_{F, z o n e}=1-\exp [-\lambda(P(g \leq 0))]
$$

which reduces to $P_{F, z o n e} \approx \lambda \cdot P(g \leq 0)$ for small values of $\lambda$.

In the context of this paper $P(g \leq 0)$ will be calculated using Monte Carlo sampling; therefore, to simplify the proceeding notation $P(g \leq 0)$ will be written $P_{M C}$, which is to represent to probability-of-fracture given a set of random variables prescribed in the limit state function. Therefore, Eq. ( 10 ) becomes

$$
P_{F, z o n e}=1-\exp \left[-\lambda \cdot P_{M C}\right]
$$

\section{B. Disk Risk}

The probability-of-fracture of the disk can be modeled as a series system of the zones:

$$
P_{F}=1-\prod_{k=1}^{n} P(\text { no failure in zone } k)=1-\prod_{k=1}^{n}\left[1-P_{F, \text { zone } k}\right]
$$

where $n$ equals the number of zones, which reduces to $P_{F} \approx \sum_{k=1}^{n} P_{F, \text { zone } k}$ for small values of $P_{F, z o n e}$.

\section{Probabilistic Sensitivities for Random Variables in DARWIN}

Probabilistic sensitivities are defined as the partial derivative of the probability-of-fracture with respect to the parameters of the random variables. In the proceeding sections, the development of the methodology for computing probabilistic sensitivities will given initially in general terms using the parameter $\theta$, where $\theta$ represents a parameter of the random variable, and then in more specific terms of the actual parameters that describe the random variables used within DARWIN.

\section{A. Sensitivity of the Probability-of-fracture of the Disk with respect to $\theta$}

The probability-of-fracture of the disk is given by Eq.( 12 ). Therefore, we compute the partial derivative of Eq. ( 12 ) with respect to the parameter $\theta$ as follows.

$$
\frac{\partial P_{F}}{\partial \theta_{j}}=\sum_{i=1}^{\hat{n}}\left[\prod_{k=1}^{n} \frac{\partial P_{F, z o n e i}}{\partial \theta_{j}} \cdot\left[\frac{\left(1-P_{F, \text { zone } k}\right)}{\left(1-P_{F, \text { zone } i}\right)}\right]\right]
$$

where $\hat{n}$ is defined as the number of zones affected by $\theta_{j}$. However, $P_{F}=\prod_{k=1}^{n}\left(1-P_{F, \text { zone } k}\right)$ and $\frac{\partial P_{F, \text { zone } i}}{\partial \theta_{j}} \cdot \frac{1}{\left(1-P_{F, \text { zone } i}\right)}$ are constant with in the product operator. As a result, Eq. ( 13 )can be simplified to the following equation: 


$$
\left.\frac{\partial P_{F}}{\partial \theta_{j}}=\sum_{i=1}^{\hat{n}}\left(1-P_{F}\right) \cdot\left(\frac{\partial P_{F, \text { zone } i}}{\partial \theta_{j}} \cdot \frac{1}{\left(1-P_{F, \text { zone } i}\right.}\right)\right)
$$

Also, $P_{F}$ is independent of $i$, therefore, Eq. ( 14 )can written

$$
\left.\frac{\partial P_{F}}{\partial \theta_{j}}=\left(1-P_{F}\right) \cdot \sum_{i=1}^{\hat{n}}\left(\frac{\partial P_{F, z \text { zon } i}}{\partial \theta_{j}} \cdot \frac{1}{\left(1-P_{F, \text { zone } i}\right.}\right)\right)
$$

\section{B. Sensitivity of the Probability-of-Fracture per Zone with respect to $\theta_{j}$}

From inspection of Eq. ( 15 ), it can be seen that the only term not determined is $\frac{\partial P_{F, z o n e} i}{\partial \theta_{j}}$. Therefore, we compute the partial derivative of Eq. ( 10 ) with respect to $\theta_{j}$.

$$
\begin{aligned}
& \frac{\partial P_{F, \text { zonei }}}{\partial \theta_{j}}=\left(\frac{\partial \lambda_{i}}{\partial \theta_{j}} \cdot P_{M C_{i}}+\lambda_{i} \cdot \frac{\partial P_{M C_{i}}}{\partial \theta_{j}}\right) \cdot \exp \left[-\lambda_{i}\left(P_{M C_{i}}\right)\right] \\
& =\left(\frac{\partial \lambda_{i}}{\partial \theta_{j}} \cdot P_{M C_{i}}+\lambda_{i} \cdot \frac{\partial P_{M C_{i}}}{\partial \theta_{j}}\right) \cdot\left(1-P_{F, \text { zone } i}\right)
\end{aligned}
$$

Inserting this result into Eq. (15) yields

$$
\frac{\partial P_{F}}{\partial \theta_{j}}=\left(1-P_{F}\right) \cdot \sum_{i=1}^{\hat{n}}\left(\frac{\partial \lambda_{i}}{\partial \theta_{j}} \cdot P_{M C_{i}}+\lambda_{i} \cdot \frac{\partial P_{M C_{i}}}{\partial \theta_{j}}\right)
$$

In Eq. ( 17 ), the term $\frac{\partial \lambda_{i}}{\partial \theta_{j}}$ is only non-zero when formulating the sensitivity with respect to $a_{\min }$ of the exceedance curve, which is a function of $\lambda_{i}$, and for all other sensitivities this term is zero.

\section{Sensitivity of the Monte Carlo Probability with respect to $\theta$}

In the scope of this paper, $P_{M C}$ is the probability of failure, given all random variables, computed using Monte Carlo sampling. In integral form, $P_{M C}$ is written

$$
P_{M C}=\int_{g \leq 0} f_{x}(\widetilde{x}) \cdot d \widetilde{x}
$$

where $\widetilde{x}$ is the vector of random variables, $f_{\widetilde{x}}(\widetilde{x})$ is the joint density function of $\tilde{x}$, and $g$ is the limit state function that defines fracture.

In order to compute the partial derivative of $P_{M C}$ with respect to some parameter of the random variables $\theta_{j}$, we take the derivative of Eq. ( 18 ) 


$$
\frac{\partial P_{M C}}{\partial \theta_{j}}=\frac{\partial}{\partial \theta_{j}} \cdot \int_{g \leq 0} f_{\widetilde{x}}(\tilde{x}) \cdot d \widetilde{x}
$$

Inserting the indicator function $I(\tilde{x})$, which is defined as equal to 1 if $g(\tilde{x}) \leq 0$ and zero otherwise,

$$
\frac{\partial P_{M C}}{\partial \theta_{j}}=\int_{-\infty}^{\infty} I(\tilde{x}) \cdot \frac{\partial f_{\widetilde{x}}(\widetilde{x})}{\partial \theta_{j}} \cdot d \widetilde{x}+\text { Boundary Term }
$$

The parameter $\theta_{j}$ is only a parameter of one of the density functions comprising the joint density for the independent random variables, the derivative only applies to that density function containing $\theta_{j}$.

$$
\begin{gathered}
\frac{\partial P_{M C}}{\partial \theta_{j}}=\int_{-\infty}^{\infty} I(\tilde{x}) \cdot \frac{\partial f_{x_{i}}\left(x_{i}\right)}{\partial \theta_{j}} \cdot \frac{1}{f_{x_{i}}\left(x_{i}\right)} \cdot f_{\widetilde{x}}(\tilde{x}) \cdot d \widetilde{x}+\text { Boundary Term } \\
=E\left[I(\tilde{x}) \cdot \frac{d f_{x_{i}}\left(x_{i}\right)}{d \theta_{j}} \cdot \frac{1}{f_{x_{i}}\left(x_{i}\right)}\right]+\text { BoundaryTerm }
\end{gathered}
$$

The boundary term arises because the derivative is being taken with respect to a parameter that is also in the limits of integration. To compute this term, we must employ the use of Leibnitz rule of integration which is given by the following equation:

$$
\frac{\partial P}{\partial \theta_{j}}=\frac{\partial}{\partial \theta_{j}} \int_{\theta_{j}}^{c} f_{x}(x) d x=\int_{\theta_{j}}^{c} \frac{\partial f_{x}(x)}{\partial \theta_{j}} d x+f(c, x) \cdot \frac{\partial c}{\partial \theta_{j}}-f\left(\theta_{j}, x\right) \cdot \frac{\partial \theta_{j}}{\partial \theta_{j}}
$$

where $c$ is a constant.

1. Sensitivity of $P_{M C}$ with respect to $a_{\min }$

It can be shown that the partial derivative of $P_{M C}$ with respect to $a_{\min }$ is as follows,

$$
\begin{aligned}
\frac{\partial P_{M C}}{\partial a_{\text {min }}} & =E[I(\tilde{x})] \cdot f_{A}\left(a_{\text {min }}\right) \\
& =P_{M C} \cdot f_{A}\left(a_{\text {min }}\right)
\end{aligned}
$$

where $f_{A}\left(a_{\min }\right)$ is the $p d f$ of the defect distribution evaluated at $a_{\min }$, and

$$
f_{A}(a)=-\frac{N\left(a_{i+1}\right)-N\left(a_{i}\right)}{a_{i+1}-a_{i}} \cdot\left(\frac{1}{N\left(a_{\min }\right)-N\left(a_{\max }\right)}\right)
$$


where $N(a)$ is the exceedance value associated with the defect size $a$ and, $N\left(a_{\min }\right)$ and $N\left(a_{\max }\right)$ are the exceedance values of the minimum and maximum defect size from the defect distribution, respectively. Eq. ( 24 ) is exact if the defect distribution is the only random variable but is a first-order estimate if there are additional random variables.

The mean occurrence rate $\lambda$ is a function of $a_{\text {min }}$ and, therefore, the derivative of $\lambda$ with respect to $a_{\min }$ is non-zero and is needed in the formulation of Eq.( 17 ). In DARWIN, $\lambda$ (or $\lambda_{i}$ per zone) is defined as follows ${ }^{3}$ :

$$
\lambda_{i}=N\left(a_{\min }\right) \cdot \frac{\Psi_{i}}{\Psi}
$$

where $N\left(a_{\min }\right)$ is the exceedance value associated with the minimum defect size from the defect distribution, $\Psi_{i}$ is the volume or surface area of zone $i$, and $\Psi$ is the reference volume or the reference surface area.

Therefore, the resulting partial derivative is

$$
\begin{aligned}
\frac{\partial\left(\lambda_{i}\right)}{\partial a_{\min }} & =\frac{\partial N\left(a_{\min }\right)}{\partial a_{\min }} \cdot \frac{\Psi_{i}}{\Psi} \\
& =-f_{A}\left(a_{\min }\right) \cdot\left[N\left(a_{\min }\right)-N\left(a_{\max }\right)\right] \cdot \frac{\Psi_{i}}{\Psi}
\end{aligned}
$$

2. Sensitivity of $P_{M C}$ with respect to $a_{\max }$

The derivation for the partial derivative of the defect distributions $p d f$ with respect to $a_{\text {max }}$ is similar to that for $a_{\min }$. When defect size is the only random variable, a variation of $a_{\max }$ modifies the domain of integration; therefore, a boundary term is required. It can be shown that the derivative is

$$
\frac{\partial P_{M C}}{\partial a_{\max }}=E[I(\tilde{x})] \cdot\left(-f_{A}\left(a_{\max }\right)\right)+f_{A}\left(a_{\max }\right)
$$

or, simply

$$
\begin{aligned}
\frac{\partial P_{M C}}{\partial a_{\max }} & =f_{A}\left(a_{\max }\right) \cdot(1-E[I(\tilde{x})]) \\
& =f_{A}\left(a_{\max }\right) \cdot\left(1-P_{M C}\right)
\end{aligned}
$$

where $f_{A}\left(a_{\max }\right)$ is the $p d f$ of the defect distribution evaluated at $a_{\max }$. It is important to point out that the boundary term presented here, $f_{A}\left(a_{\max }\right)$, is exact if the exceedance curve is the only random variable. If there are additional random variables, Eq. ( 29 )is a first order estimate.

\section{Sensitivity of $P_{M C}$ with respect to cov}

The following derivation applies equally to the Stress Multiplier and Life Scatter variables both variables are modeled using the lognormal distribution. 
The probability density function for the lognormal distribution in terms of the median, $\widetilde{X}$, and the coefficient of variation, cov, is:

$$
f(x)=\frac{1}{x \sqrt{2 \pi \cdot \ln \left(1+\operatorname{cov}^{2}\right)}} \cdot \exp \left[-\frac{(\ln (x)-\ln (\tilde{X})}{2 \cdot \ln \left(1+\operatorname{cov}^{2}\right)}\right]
$$

Per Eq. ( 22 ), the resulting sensitivity of $P_{M C}$ with respect to the cov is:

$$
\frac{\partial P_{M C}}{\partial \operatorname{cov}}=E\left[I(\tilde{x}) \cdot \frac{\operatorname{cov} \cdot\left(-\ln \left(1+\operatorname{cov}^{2}\right)+(\ln (\tilde{X})-\ln (x))^{2}\right)}{\left(1+\operatorname{cov}^{2}\right) \cdot \ln \left(1+\operatorname{cov}^{2}\right)^{2}}\right]
$$

It should be noted that because the cov is not a bound of the integration domain, a boundary term is not needed.

4. Sensitivity of $P_{M C}$ with respect to $\tilde{X}$

As in the above case, the following derivation applies equally to the Stress Multiplier and Life Scatter variables. The resulting expression is as follows:

$$
\frac{\partial P_{M C}}{\partial \widetilde{X}}=E\left[I(\tilde{x}) \cdot \frac{\ln (x)-\ln (\tilde{X})}{\widetilde{X} \cdot \ln \left(1+\operatorname{cov}^{2}\right)}\right]
$$

\section{Application}

Example \#1. Initial Defect Size as Only Random Variable.

The model for this example consists of the titanium ring outlined by advisory circular AC-33.14- $1^{9}$ subjected to centrifugal loading as shown in Figure 1.

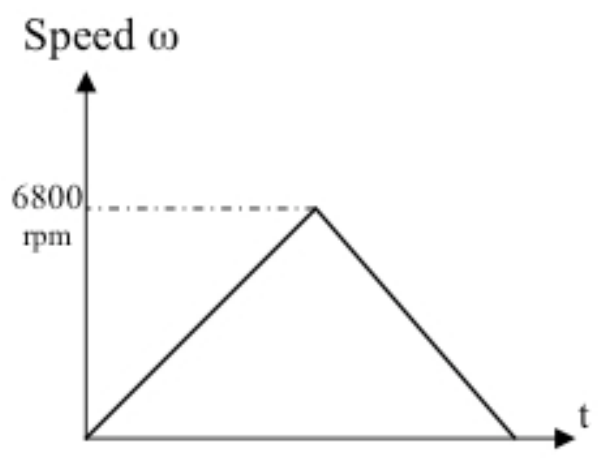

Figure 1. Loading cycle for ring.

The titanium ring has a rectangular cross-section and is under rotation with an external load. The crack grows under cyclical loading until failure - defined when the stress intensity factor exceeds the fracture toughness. 
For this problem, the titanium ring cross-section has been divided into 24 zones, of approximately equal risk as shown in Figure 3 where only half the cross-section is modeled due to symmetry. The exceedance curve used for generating defect samples has been obtained from the DARWIN defect library.

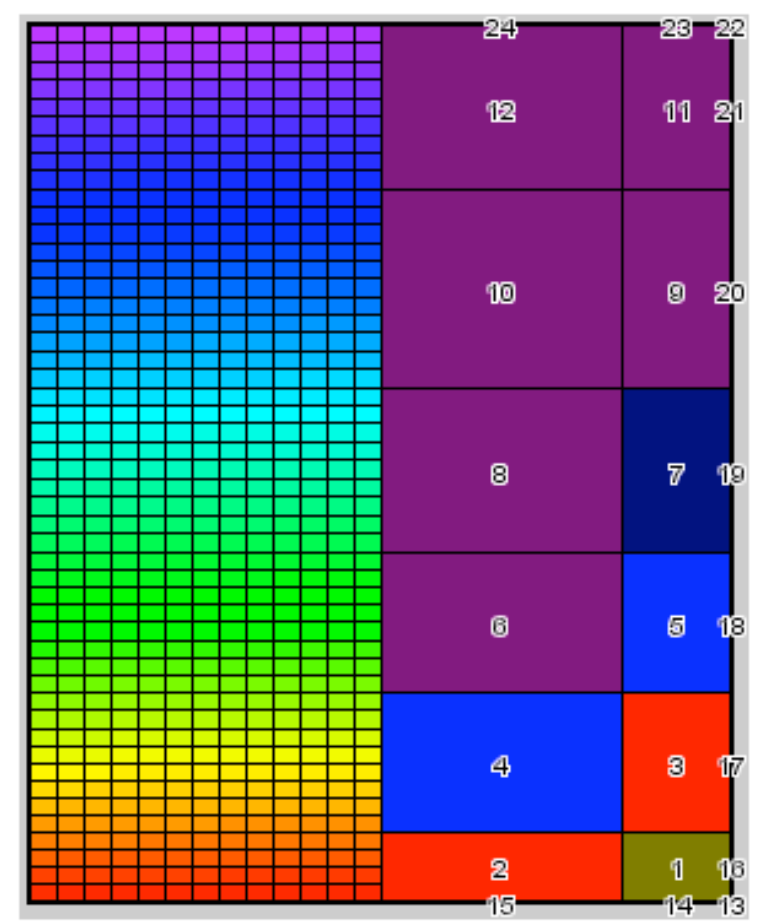

Figure 3. Titanium ring discretized into 24 zones.

From Table 2, it is quite evident that the results from the sampling methodology compare extremely well with the results from the finite difference approach. In fact, these results support the equations for sensitivities to the bounds of the exceedance curve, developed here, and the need for a boundary term for the sensitivity with respect to $a_{\max }$. The results in Table 2 also provide a measure of how well the exceedance curve used for this problem was constructed. Because the sensitivities to the bounds of the exceedance curve are extremely small relative to the probability-of-fracture of the disk, it can be said that for this problem there is no need to construct another exceedance curve to encapsulate a wider range in defect sizes.

Table 2. Comparison of Results for Example 1 from Sampling and Finite Difference Techniques.

\begin{tabular}{|c|c|c|}
\hline Random Variables & Sampling Technique & Finite Difference Technique \\
\hline$\frac{\partial P_{f}}{\partial a_{\min }}$ & $8.4047 \mathrm{E}-10$ & $8.3033 \mathrm{E}-10$ \\
\hline$\frac{\partial P_{f}}{\partial a_{\max }}$ & $6.0010 \mathrm{E}-12$ & $5.9921 \mathrm{E}-12$ \\
\hline
\end{tabular}




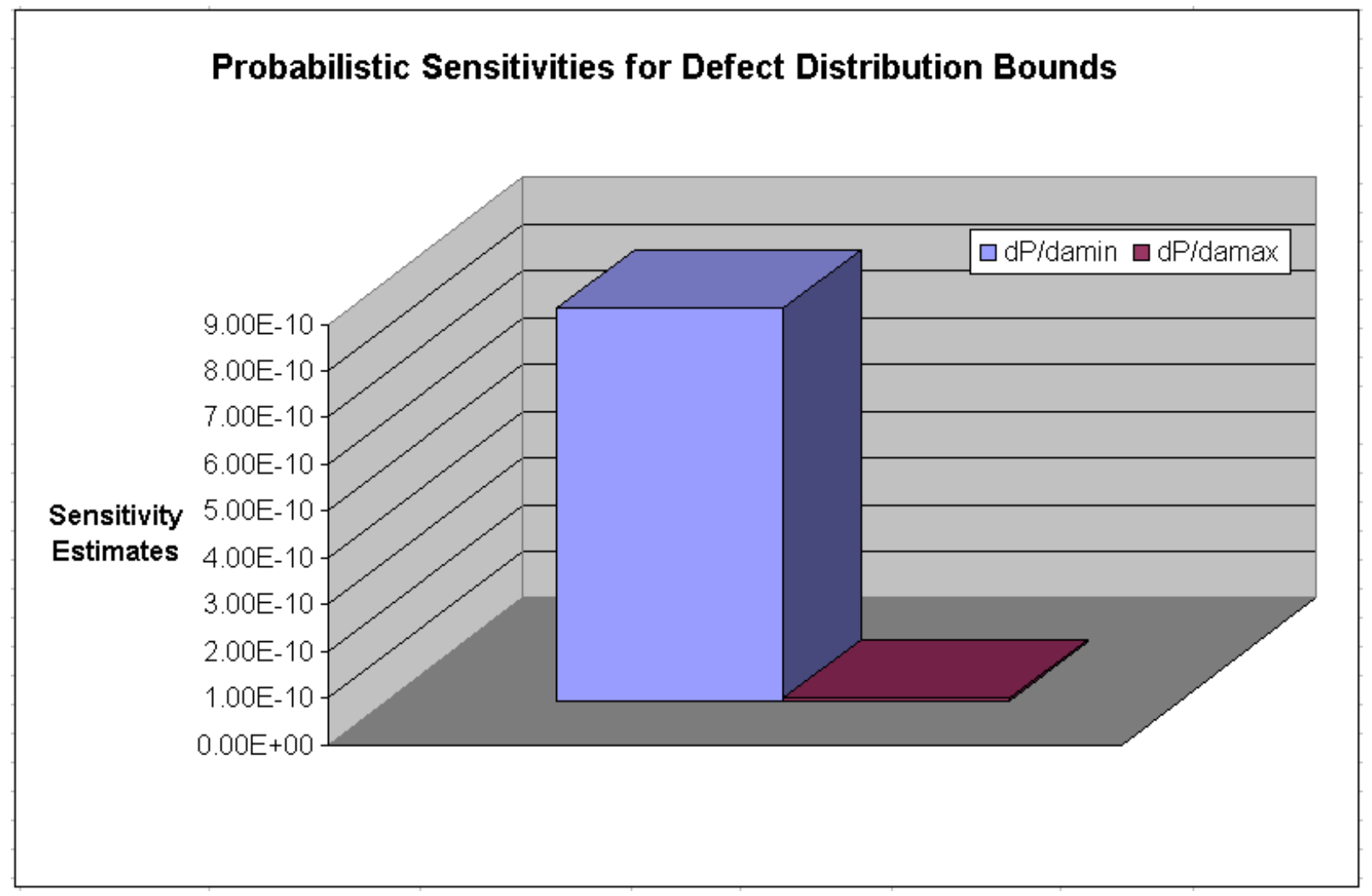

Figure 5. Sensitivities for the bounds of the defect distribution.

Example \#2. Life Scatter, Stress Multiplier, and Defect Size as Random Variables

In this example, the random variables Life Scatter and Stress Multiplier have been added to example problem 1, each with a median value of 1 and coefficient of variation of 0.1 . The analysis was performed by computing the sensitivities according to Eq. ( 17 ), with Eqs. ( 24 ), ( 27 ), (29), ( 31 ), and ( 32 ), then compared to those a numerical finite difference calculation computed from successive DARWIN runs. The results of this study are summarized in Figure 6 and Table 3 below.

Defect Distribution.

Table 3. Comparison of Results for Example 2 from Sampling and Finite Difference Techniques using 100,000 Monte Carlo samples.

\begin{tabular}{|c|c|c|}
\hline Random Variables & Sampling Technique & Finite Difference Technique \\
\hline$\frac{\partial P_{f}}{\partial S M_{C O V}}$ & $7.802050 \mathrm{E}-4$ & $7.901650 \mathrm{E}-4$ \\
\hline$\frac{\partial P_{f}}{\partial S M_{\text {median }}}$ & $1.040530 \mathrm{E}-3$ & $1.056080 \mathrm{E}-3$ \\
\hline$\frac{\partial P_{f}}{\partial L S_{C O V}}$ & $4.745940 \mathrm{E}-5$ & $5.044580 \mathrm{E}-5$ \\
\hline
\end{tabular}




\begin{tabular}{|c|c|c|}
\hline$\frac{\partial P_{f}}{\partial L S_{\text {median }}}$ & $-2.556550 \mathrm{E}-4$ & $-2.224830 \mathrm{E}-4$ \\
\hline$\frac{\partial P_{f}}{\partial a_{\min }}$ & $1.148740 \mathrm{E}-9$ & $2.72167 \mathrm{E}-8$ \\
\hline$\frac{\partial P_{f}}{\partial a_{\max }}$ & $5.988860 \mathrm{E}-12$ & $3.180280 \mathrm{E}-10$ \\
\hline
\end{tabular}

\section{Probabilistic Sensitivities for Random Variables in DARWIN}

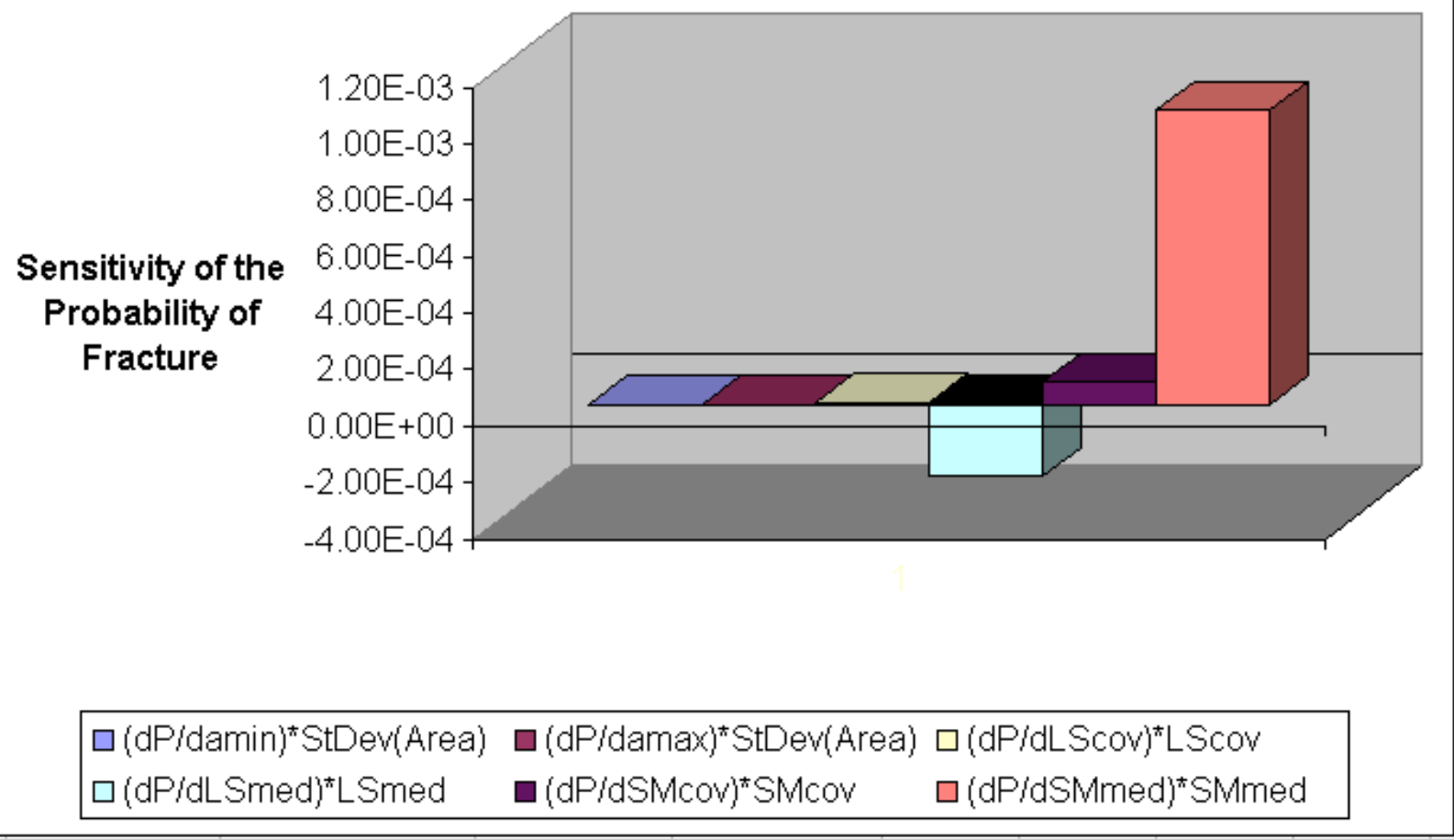

Figure 6. Non-dimensionalized sensitivities for parameters of random variables Life Scatter, Stress Multiplier, and Defect Distribution.

The results in Figure 6 indicate that the most important variable in the model is the Stress Multiplying factor. The results in Table 3 for sensitivities to both $a_{\min }$ and $a_{\max }$ do not agree well with the results from finite difference. The reason for the disparity in the estimates can be attributed to the fact that the sensitivity equations for $a_{\min }$ and $a_{\max }$ are exact in one-dimension and do not take into account the interaction between variables in higher dimensions and that the order of the estimates are such that it is difficult to obtain good accuracy using finite difference. For example, small differences in the computed probability-of-fracture can be overridden by sampling error. However, these first-order sensitivity estimates do provide an order-of-magnitude estimate useful for comparison purposes. 


\section{Conclusion}

The ability to quantify how the probability-of-fracture is effected by changes to distribution parameters that characterize the random variables is, undeniably, very valuable for design and analysis. The information can be used to determine the importance of a random variable. A methodology is presented and demonstrated that determines the sensitivities of the probability-of-fracture of a zone-based fatigue analysis with respect to the parameters of the

probability distributions of the input random variables. The sensitivities are computed without additional limit state calculations. The methodology is demonstrated using a probabilistic fatigue analysis of a gas turbine compressor disk.

\section{Acknowledgements}

This work was supported in part by the Federal Aviation Administration under Cooperative Agreement 95-G041 and Grant 99-G-016. The authors wish to thank the FAA Technical Center project managers, Bruce Fenton and Joe Wilson for their continued diligence and encouragement and Tim Mouzakis of the FAA Engine and Propeller Directorate for his continued support. The ongoing contributions of the project Steering Committee from Pratt \& Whitney, Honeywell, General Electric and Rolls-Royce are also gratefully acknowledged.

\section{References}

${ }^{1}$ Millwater, H.R., Wu, Y.-T.,Enright, M. P., Riha, D., Leverant, G., McCliung, C., Chell, G., Kuhlman, C., Fitch, S. Gorelik, M., Herrman, D., Mihlbachler, G., Lehmann, D. (1999). "A probabilistically-based damage tolerance analysis computer program for hard alpha defects in titanium rotors," USAF Structural Integrity Program Conference, San Antonio, TX, Nov. 30.

${ }^{2}$ Leverant, G.R., Millwater, H. R., Littlefield, D. L., Wu, Y.-T., McClung, R. C.. (1997). "A probabilistic approach to aircraft turbine rotor material design," ASME International Gas Turbine \& Aeroengine Congress ,Paper 97-GT-22

${ }^{3}$ G.R. Leverant, H.R. Millwater, R.C. McClung, M.P. Enright, "A New Tool for Design and Certification of Aircraft Turbine Rotors,” Journal of Engineering for Gas Turbines and Power, Vol. 126, No. 1, pp. 155-159, 2004

${ }^{4}$ H. Millwater, S. Fitch, J. Wu, D. Riha, M. Enright, G. Leverant, C. McClung, C. Kuhlman, G. Chell, Y.-D. Lee, “A Probabilistically-based Damage Tolerance Analysis Computer Program for Hard Alpha Anomalies in Titanium Rotors," ASME International Gas Turbine Conference (IGTI), ASME Paper No. 2000-GT-0421, Munich, Germany, May 8-11, 2000

${ }^{5}$ Millwater, H.R. Enright, M. P., Fitch, S. (2002). "A convergent probabilistic technique for risk assessment of gas turbine disks subject to metallurgical defects," Proceedings of the 43rd AIAA/ASME/ASCE/AHS/ASC Structures, Structural Dynamics, and Materials Conference, Non-Deterministic Approaches Forum, Denver, CO, April 22-25.

${ }^{6}$ Enright, M.P., Huyse, L., McClung, R.C. (2005) "Fracture Mechanics-based Probabilistic Life Prediction of Components with Large Numbers of Inherent Material Anomalies”, ICOSSAR Conference, June 2005.

${ }^{7}$ A.K. Karamchandani. New approaches to structural system reliability, Ph.D. thesis, Dept. of Civil Engineering, Stanford University, 1990.

${ }^{8}$ Wu, Y.-T. (1994). "Computational Methods for Efficient Structural Reliability and Reliability Sensitivity Analysis", AIAA Journal, 32 (8), 1717-1723

${ }^{9}$ Federal Aviation Administration (2001). "Advisory Circular - Damage Tolerance for High Energy Turbine Engine Rotors". U.S. Dept. of Transportation, AC 33.14-1, Washington, DC. 